\title{
Antimicrobial Resistance - Emerging trends and the need for Antibiotic policy
}

With the discovery of Penicillin in 1929 by Alexander Fleming and subsequent use in clinical practice, we were all elated at the thought of having a magic bullet by which we can win the battle against microbes but before the battle-field could be cleared of the debris, there appeared a new strain of microbe against which the prevailing antibiotic became ineffective. Research for newer antibiotic with increased killing power is still going on but we have to remember that bacteria is a very versatile organism. Whatever mechanisms we device to kill it, it develops newer mechanisms for its survival. This constant battle between Man and Bacteria i.e. the antimicrobial resistance has become so alarming that WHO has adopted this year the slogan of Antimicrobial resistance as its theme for this years' World Health Day on $7^{\text {th }}$ April 2011. Awareness about the injudicious use of antimicrobials which can create a selection pressure on microbes resulting in emergence of new resistant strains even to the most advanced drugs such as Imipenam and Vancomycin. These multidrug resistant bacteria roam about in the normal flora of human being and when a pathogenic bacteria enters the same individual, the resistance mechanisms are transferred to the pathogen resulting in treatment failure with the consequent increase in morbidity and mortality. Moreover the individual who is carrying the multi-drug resistant bacteria as a part of normal flora has the potential to disperse the same organism to another patient. This happens mostly by the nurses, doctors and other staffs of wards, ICU's or even by the visitors of a hospital. Effective Infection Control measures has become an essential part of a hospital program.

Mechanisms by which a bacteria becomes resistant to drugs such as alteration of targets, alteration of metabolic pathways, alteration of permeability of drugs are numerous but the most common resistance mechanism is the production of the enzyme $\beta$-lactamases. It has been developed by most of the both Gram negative and Gram positive pathogenic microbes. These enzymes split the $\beta$-lactam ring containing antimicrobials such as the Penicillins, Amoxycillins and $1^{\text {st }}$ and $2^{\text {nd }}$ generation Cephalosporins and make them ineffective. Now-a-days another more potent enzyme, the Extended-spectrum $\beta$-lactamases are being produced by many strains of E.coli, Klebsiella, Pseudomonas and Acinetobacter thus destroying the $3^{\text {rd }}$ or even the $4^{\text {th }}$ generation Cephalosporins. Majority of Fluoroquinolones have become ineffective as resistant bacteria have altered their DNA gyrases. At times now-a-days, we are left with only a few highly costly drugs such as the Carbapenams -Imipenam and the Meropenam to make our last ditch fight against the pathogens. Alas, few microbes have developed resistance to the Carbapenams also. This unimaginable picture of all the microbes becoming resistant to all the available antibiotic is not far-away considering the way in which antibiotics are being dispensed in our country. It is available over the counter in a Pharmacy to anyone who has the capacity to buy it. Patients suffering from non bacterial infections are being sold antibiotics without any prescriptions by compounders, pharmacist and even salesman thus selecting a resistant bacterial population in the normal flora. Even lot of doctors do not have the time to think about the etiology of the syndrome and prescribe injudiciously higher antibiotics. Cefexime is being prescribed in Staphylococcal infection while Ceftriaxone in Pseudomonas infections. Even Ciprofloxacin is being prescribed for Sore throat. This injudicious use of antimicrobials should be stopped as early as possible. Over the counter sale of Antibiotics should be banned by the Government at once. Each and every hospital should have their own antibiotic policy. General practitioners should have a more rational approach to prescribing antibiotics. Without Culture \& Sensitivity reports, use of higher antibiotics should be restricted. Time has come 
for some drastic measures to be taken before it is too late.

Infection Control programs if implemented properly in a hospital can go a lot in preventing the emergence and spread of resistant bacteria. Here we would like to mention the name of our deceased Director and later acting Principal Prof. M.R. Azad who breathed his last on the night of $25^{\text {th }}$ March, 2011(Inalilahe-waInarajeoun). His futuristic vision of setting up of a Infection Control program in Dhaka National Medical College Hospital way back in 2005 was an initial step in the right direction. This program started with the simple practice of hand washing and collection, decontamination, safe disposable of syringes but alas due to bureaucratic tangles it is still in its infant stages. Without a proper support from the management, climbing the ladder of infection control is very difficult.

Prof. M. A. Basher

Prof. Munir Hassan 